\title{
Management of Complex Weed Flora through Herbicide Combinations in Irrigated Wheat (Triticum aestivum L.).
}

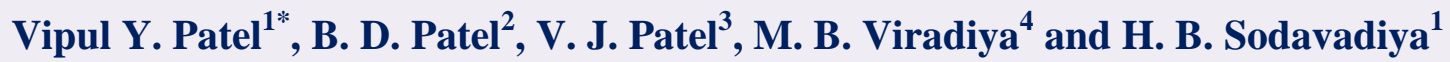 \\ ${ }^{1}$ Department of Agronomy, ${ }^{2}$ Agronomist \& PI, AICRP Weed Management, ${ }^{4}$ Department of \\ Agril.Chem. \& Soil Sci., BACA, AAU, Anand, Gujarat, India \\ ${ }^{3}$ Sheth M. C. Polytechnic in Agriculture, \\ *Corresponding author
}

Keywords

Pre/tank mix, Weed Control Efficiency, Weed Index, Yield, $\mathrm{B}: \mathrm{C}$ ratio

Article Info

Accepted:

20 December 2020

Available Online:

10 January 2021
A field experiment was conducted during rabi season of the year 2017-18 and 2018-19 on loamy sand soil at the farm of AICRP-Weed Management, Anand Agricultural University (Gujarat) to study the management of complex weed flora through herbicide combinations in irrigated wheat (Triticum aestivum L.). The experiment comprised of twelve treatments tested in randomized block design with three replications. Findings of the experiment clearly showed that, among all the treatments significantly lower weed density, weed dry weight and weed index as well as higher grain yield, straw yield and B:C ratio was achieved with post emergence application of pre-mix clodinafop + metsulfuron $60+4 \mathrm{~g} / \mathrm{ha}$ followed by sulfosulfuron + metsulfuron $30+2 \mathrm{~g} / \mathrm{ha}$ and mesosulfuron + iodosulfuron $12+$ $2.4 \mathrm{~g} / \mathrm{ha}$.

\section{Introduction}

Wheat (Triticum aestivum L.) is one of the most important staple food crop cultivated in almost all countries in the world. One of major rabi crops cultivated in India is wheat specially north Indian plains and north west Himalayas, its like a backbone of food security of nation as evident from its contribution to nearly one third of total food grain productions. The short stature of new dwarf varieties coupled with higher fertilizers and irrigation requirements creates favourable ecological conditions for weed growth.
Weeds compete with crop for sunlight, space, nutrients and moisture. In wheat the earlier period up to 30-40 days is considered as critical crop weed competition it is one of the major factors responsible for low productivity of wheat. Up to $66 \%$ wheat grain yield reduce due to uncontrolled weeds which was reported by Angiras et al., (2008). The reduction in yield may be due to infestation of some dominated weed flora at early growth stage and the luxuriant growth of weeds because of frequent irrigation and dominance of monotony cropping system. Weed management is one of the major input costs of 
production. Mechanical and hand weeding methods are laborious and time consuming besides, weeds with similar morphological characters like crops are likely to be escaped. Additionally, by the time weeds can be removed by manually, they have already competed with the crop for necessary requirements and ultimately reduce the yield. Under this situation, the chemical weed control is found to be effective and economical alternate. Herbicides play an important role for weed control in close spaced crops like wheat and barley, where manual or mechanical weeding is difficult to control the mimicry weeds. However, continues and heavy reliance on herbicides creates an environment favourable for weed resistance to herbicides, weed population shifts and off-site movements of herbicides (Rao and Nagmani, 2010).

However, application of herbicides in combination either pre-plant incorporation or pre-emergence or post-emergence generally increase the spectrum of weed control or the length of residual weed control. Tank-mixing of herbicides may improve the spectrum of weeds controlled in a single application which saves the time and labour in weed management programme. Mixing compatible herbicides from different chemical families may improve control of specific weed populations.

Herbicide combinations may also provide control of several weed types at the same time, such as grassy and broadleaf weeds. Further, herbicide combination is better because of low rates required with minimum herbicide residue in soil which will biodegrade in a shorter time and improve the succeeding crop safety. It enhanced activity on target weed species as well as decreased toxicity on crops and improves the herbicide efficacy hence, the present study was undertaken.

\section{Materials and Methods}

Field experiment was carried out during rabi season of the year 2017-18 and 2018-19 on loamy sand soil at the farm of AICRP-Weed Management, B. A. College of Agriculture, Anand Agricultural University, Anand (Gujarat). The experiment was laid out in randomized block design with twelve treatments viz., $\mathrm{T}_{1}$ : Pendimethalin PE 750 $\mathrm{g} / \mathrm{ha}, \mathrm{T}_{2}$ : Pendimethalin + metribuzin (Tank mix) $\mathrm{PE}(500+175 \mathrm{~g} / \mathrm{ha}), \mathrm{T}_{3}$ : Pendimethalin (PE) $500 \mathrm{~g} / \mathrm{ha} f b$ sulfosulfuron (PoE) $18 \mathrm{~g} / \mathrm{ha}$ $\left(\mathrm{T}_{3}\right), \mathrm{T}_{4}$ : Sulfosulfuron PoE (25 g/ha), $\mathrm{T}_{5}$ : Sulfosulfuron + 2,4-D (SS) (Tank mix) PoE $(25+250 \mathrm{~g} / \mathrm{ha}), \mathrm{T}_{6}:$ Sulfosulfuron + metsulfuron (Premix) PoE $(30+2 \mathrm{~g} / \mathrm{ha}), \mathrm{T}_{7}$ : Clodinafop PoE (60 g/ha), T : Clodinafop + metsulfuron (Premix) PoE $(60+4 \mathrm{~g} / \mathrm{ha}), \mathrm{T}_{9}$ : Metsulfuron methyl PoE (4 g/ha), $\mathrm{T}_{10}$ : Mesosulfuron + iodosulfuron (Premix) PoE $(12+2.4 \mathrm{~g} / \mathrm{ha}), \mathrm{T}_{11}$ : Hand weeding (20 and 40 DAS) and $T_{12}$ : Un-weeded control and replicated three times in rabi season during the years 2017-18 and 2018-19. Recommended seed rate i.e. $120 \mathrm{~kg} / \mathrm{ha}$ of wheat cv. "Gujarat wheat 451" was manually sown keeping the row distance $22.5 \mathrm{~cm}$ during second fortnight of November during both the years. The first irrigation was given immediately after sowing for uniform germination of seeds and remaining irrigations were applied as per the requirement. For good take care of crop all the recommended practices are adopted. Pre emergence herbicides were applied after next day of first irrigation and post-emergence herbicides were applied at 25-30 DAS with knapsack sprayer fitted with flat fan nozzle by using 500 litre of water/ha. Weed parameters measure periodically from net plot area with using $0.25 \mathrm{~m}^{2}$ quadrant, later on it was converted into $\mathrm{m}^{2}$ area. Square root transformation was use for the analysis of weed density and dry weight of weed. 


\section{Results and Discussion}

\section{Effect on weed}

Data reported in Table 1 and 2 indicated that post emergence application of premix herbicide sulfosulfuron + metsulfuron $30+2$ $\mathrm{g} / \mathrm{ha}\left(\mathrm{T}_{6}\right)$, clodinafop + metsulfuron $60+4$ $\mathrm{g} / \mathrm{ha}\left(\mathrm{T}_{8}\right)$ and mesosulfuron + iodosulfuron 12 $+2.4 \mathrm{~g} / \mathrm{ha}\left(\mathrm{T}_{10}\right)$ provide complete control of weeds at 40,80 DAS and at harvest hence, no weed dry weight was recorded under these treatments. While application of sulfosulfuron $+2,4-\mathrm{D}(\mathrm{SS})(\mathrm{PoE}) 25+250 \mathrm{~g} / \mathrm{ha}\left(\mathrm{T}_{5}\right)$ performed better followed by application of pendimethalin (PE) $500 \mathrm{~g} / \mathrm{ha} f b$ sulfosulfuron $(\mathrm{PoE}) 18 \mathrm{~g} / \mathrm{ha}\left(\mathrm{T}_{3}\right)$, hand weeding at 20 and 40 DAS $\left(\mathrm{T}_{11}\right)$ and sulfosulfuron (PoE) $25 \mathrm{~g} / \mathrm{ha}$ $\left(\mathrm{T}_{4}\right)$ during both years. Monocot weeds were effectively control by sulfosulfuron, clodinafop and mesosulfuron methyl while 2,4-D, metsulfuron and iodosulfuron proved effective against dicot weed. This indicates that herbicide molecule when mixed like; sulfosulfuron + 2,4-D (SS), sulfosulfuron + metsulfuron, clodinafop + metsulfuronand mesosulfuron + iodosulfuron became more effective against both monocot and dicot weed flora at a same time due to better compatibility of mixture. Herbicide mixture is readily absorbed by roots, shoots of plants and translocated apoplastically and symplastically to meristemic regions, the symptoms of injury develop slowly after their application and it often takes three weeks for susceptible plants to die (Yadav, 2017). Hence, pre-mix formulations of herbicide effectively manage the both group of weeds due to their higher efficacy as compared to sole application of herbicide. These line of results are in accordance with the findings of Chaudhari et al., (2017) and Patel et al., (2017). The highest numbers of total weeds and dry weight of weeds was observed under un-weeded control $\left(\mathrm{T}_{12}\right)$ during the year 2017 18 and 2018-19 as well as in pooled analysis.
Calculation of weed control efficiency on the basis of total dry weight of weeds recorded at at harvest indicated that maximum weed control efficiency $(100 \%)$ was achieved under application of sulfosulfuron + metsulfuron $(\mathrm{PoE}) 30+2 \mathrm{~g} / \mathrm{ha}\left(\mathrm{T}_{6}\right)$, clodinafop + metsulfuron $(\mathrm{PoE}) 60+4 \mathrm{~g} / \mathrm{ha}\left(\mathrm{T}_{8}\right)$ and mesosulfuron + iodosulfuron $(\mathrm{PoE}) 12+2.4$ $\mathrm{g} / \mathrm{ha}\left(\mathrm{T}_{10}\right)$ as compared to rest of the treatment tried in the experiment. Application of metsulfuron methyl (PoE) 4 g/ha $\quad\left(\mathrm{T}_{9}\right)$ recorded lower weed control efficiency which was nearly followed by application of pendimethalin $(\mathrm{PE}) 750 \mathrm{~g} / \mathrm{ha}\left(\mathrm{T}_{1}\right)$ at $80 \mathrm{DAS}$ and at harvest. The lower weed control efficiency under said treatment might be due to minimum weed control efficacy owing to uncontrolled conditions favoured luxurious weed growth leading to increased weed dry matter therefore, weed control efficiency was decline. Higher weed control efficiency was noted with application of sulfosulfuron $+2,4-$ $\mathrm{D}(\mathrm{SS})(\mathrm{PoE}) 25+250 \mathrm{~g} / \mathrm{ha}\left(\mathrm{T}_{5}\right)$ followed by application of pendimethalin (PE) $500 \mathrm{~g} / \mathrm{ha} f b$ sulfosulfuron (PoE) $18 \mathrm{~g} / \mathrm{ha}\left(\mathrm{T}_{3}\right)$ and hand weeding at 20 and 40 DAS $\left(\mathrm{T}_{11}\right)$. The welldeveloped crop canopy, intercepting solar energy effectively covered the ground area which reduced the weed emergence and increased the weed control efficacy. Similar results were also reported by Patel et al., (2017) and Chaudhari et al., (2017).

\section{Effect on crop and economics}

Result in respect of grain and straw yields of wheat (Table 3) indicated that application of clodinafop + metsulfuron $(\mathrm{PoE}) 60+4 \mathrm{~g} / \mathrm{ha}$ $\left(\mathrm{T}_{8}\right)$ and sulfosulfuron + metsulfuron $(\mathrm{PoE})$ $30+2 \mathrm{~g} / \mathrm{ha}\left(\mathrm{T}_{6}\right)$ recorded significantly higher grain and straw yields of wheat as compared to all other treatments except application of pendimethalin (PE) $500 \mathrm{~g} / \mathrm{ha} f b$ sulfosulfuron (PoE) $18 \mathrm{~g} / \mathrm{ha}\left(\mathrm{T}_{3}\right)$, hand weeding at 20 and 40 DAS $\left(\mathrm{T}_{11}\right.$ ), sulfosulfuron + 2,4-D SS (PoE) 25 $+250 \mathrm{~g} / \mathrm{ha}\left(\mathrm{T}_{5}\right)$ and mesosulfuron + 
iodosulfuron (PoE) $12+2.4 \mathrm{~g} / \mathrm{ha} \quad\left(\mathrm{T}_{10}\right)$ (Bharat and Kachroo, 2010 and Patel et al., 2017). The higher yield under said treatment might be due to effective weed control kept the crop almost weed free during entire crop growth period that markedly reduced the competition for the moisture, space, nutrients, light that led to enhanced crop growth by utilizing greater moisture and nutrients from soil layers (Yadav 2017).

Table.1 Density of total weed as influenced by various weed management practices

\begin{tabular}{|c|c|c|c|c|c|c|c|c|c|}
\hline \multirow[t]{3}{*}{ Treatment } & \multicolumn{9}{|c|}{ Density of total weed $\left(\mathrm{no} . / \mathrm{m}^{2}\right)$} \\
\hline & \multicolumn{3}{|c|}{ At 40 DAS } & \multicolumn{3}{|c|}{ At 80 DAS } & \multicolumn{3}{|c|}{ At harvest } \\
\hline & 2017-18 & 2018-19 & Pooled & 2017-18 & 2018-19 & Pooled & 2017-18 & 2018-19 & Pooled \\
\hline \multirow{2}{*}{$\mathbf{T}_{1}$} & $5.26^{\mathrm{d}}$ & $6.18^{\mathrm{c}}$ & $5.72^{\mathrm{d}}$ & $5.49^{\mathrm{d}}$ & $6.60^{\mathrm{cd}}$ & $6.04^{\mathrm{d}}$ & $5.62^{\mathrm{cd}}$ & $6.40^{\mathrm{c}}$ & $6.01^{\mathrm{cd}}$ \\
\hline & (26.67) & (37.33) & $(32.00)$ & (29.33) & $(42.67)$ & $(36.00)$ & $(30.67)$ & $(40.00)$ & $(35.33)$ \\
\hline \multirow{2}{*}{$\mathbf{T}_{2}$} & $5.00^{\mathrm{d}}$ & $5.96^{\mathrm{c}}$ & $5.48^{\mathrm{de}}$ & $5.22^{\mathrm{de}}$ & $5.80^{\mathrm{de}}$ & $5.51^{\mathrm{d}}$ & $4.86^{\mathrm{de}}$ & $6.26^{c}$ & $5.56^{\mathrm{de}}$ \\
\hline & $(24.00)$ & $(34.67)$ & (29.33) & (26.67) & $(33.33)$ & $(30.00)$ & (22.67) & $(38.67)$ & $(30.67)$ \\
\hline \multirow{2}{*}{$\mathbf{T}_{3}$} & $4.57^{\mathrm{de}}$ & $4.72^{\mathrm{d}}$ & $4.65^{\mathrm{g}}$ & $3.93^{\mathrm{fg}}$ & $4.28^{\mathrm{fg}}$ & $4.10^{\mathrm{ef}}$ & $3.00^{\mathrm{g}}$ & $3.61^{\mathrm{e}}$ & $3.30^{\mathrm{g}}$ \\
\hline & $(20.00)$ & (21.33) & (20.67) & (14.67) & (17.33) & $(16.00)$ & $(8.00)$ & $(12.00)$ & $(10.00)$ \\
\hline \multirow{2}{*}{$\mathbf{T}_{4}$} & $5.36^{\mathrm{d}}$ & $6.36^{\mathrm{c}}$ & $5.86^{\mathrm{d}}$ & $4.96^{\text {def }}$ & $5.97^{\mathrm{de}}$ & $5.46^{\mathrm{d}}$ & $4.72^{\mathrm{ef}}$ & $5.18^{\mathrm{d}}$ & $4.95^{\mathrm{e}}$ \\
\hline & $(28.00)$ & $(40.00)$ & $(34.00)$ & $(24.00)$ & $(34.67)$ & (29.33) & $(21.33)$ & (26.67) & $(24.00)$ \\
\hline \multirow{2}{*}{$\mathbf{T}_{5}$} & $3.78^{\mathrm{e}}$ & $4.24^{\mathrm{d}}$ & $4.01^{\mathrm{g}}$ & $3.20^{\mathrm{g}}$ & $3.78^{\mathrm{g}}$ & $3.49^{f}$ & $2.49^{\mathrm{g}}$ & $3.00^{\mathrm{e}}$ & $2.75^{\mathrm{g}}$ \\
\hline & (13.33) & (17.33) & (15.33) & $(9.33)$ & (13.33) & (11.33) & (5.33) & $(8.00)$ & $(6.67)$ \\
\hline \multirow{2}{*}{$T_{6}$} & $1.00^{\mathrm{f}}$ & $1.00^{\mathrm{e}}$ & $1.00^{\mathrm{h}}$ & $1.00^{\mathrm{h}}$ & $1.00^{\mathrm{h}}$ & $1.00^{\mathrm{g}}$ & $1.00^{\mathrm{h}}$ & $1.00^{f}$ & $1.00^{\mathrm{h}}$ \\
\hline & $(0.00)$ & $(0.00)$ & $(0.00)$ & $(0.00)$ & $(0.00)$ & $(0.00)$ & $(0.00)$ & $(0.00)$ & $(0.00)$ \\
\hline \multirow[t]{2}{*}{$\mathbf{T}_{7}$} & $9.06^{\mathrm{b}}$ & $9.28^{\mathrm{b}}$ & $9.17^{\mathrm{b}}$ & $7.71^{b}$ & $8.05^{b}$ & $7.88^{\mathrm{b}}$ & $7.72^{\mathrm{b}}$ & $8.46^{\mathrm{b}}$ & $8.09^{\mathrm{b}}$ \\
\hline & $(81.33)$ & $(85.33)$ & $(83.33)$ & $(58.67)$ & $(64.00)$ & $(61.33)$ & $(58.67)$ & (70.67) & $(64.67)$ \\
\hline \multirow[t]{2}{*}{$\mathbf{T}_{8}$} & $1.00^{f}$ & $1.00^{f}$ & $1.00^{\mathrm{h}}$ & $1.00^{\mathrm{h}}$ & $1.00^{\mathrm{h}}$ & $1.00^{\mathrm{g}}$ & $1.00^{\mathrm{h}}$ & $1.00^{f}$ & $1.00^{\mathrm{h}}$ \\
\hline & $(0.00)$ & $(0.00)$ & $(0.00)$ & $(0.00)$ & $(0.00)$ & $(0.00)$ & $(0.00)$ & $(0.00)$ & $(0.00)$ \\
\hline \multirow[t]{2}{*}{$\mathbf{T}_{9}$} & $7.90^{\mathrm{c}}$ & $8.44^{\mathrm{b}}$ & $8.17^{\mathrm{c}}$ & $6.58^{\mathrm{c}}$ & $7.45^{\mathrm{bc}}$ & $7.01^{\mathrm{c}}$ & $6.25^{\mathrm{c}}$ & $6.89^{c}$ & $6.57^{c}$ \\
\hline & (62.67) & (70.67) & (66.67) & (42.67) & (54.67) & (48.67) & (38.67) & (46.67) & (42.67) \\
\hline \multirow[t]{2}{*}{$T_{10}$} & $1.00^{f}$ & $1.00^{\mathrm{e}}$ & $1.00^{\mathrm{h}}$ & $1.00^{\mathrm{h}}$ & $1.00^{\mathrm{h}}$ & $1.00^{\mathrm{g}}$ & $1.00^{\mathrm{h}}$ & $1.00^{\mathrm{f}}$ & $1.00^{\mathrm{h}}$ \\
\hline & $(0.00)$ & $(0.00)$ & $(0.00)$ & $(0.00)$ & $(0.00)$ & $(0.00)$ & $(0.00)$ & $(0.00)$ & $(0.00)$ \\
\hline \multirow[t]{2}{*}{$T_{11}$} & $5.11^{d}$ & $5.74^{\mathrm{c}}$ & $5.43^{\text {def }}$ & $4.28^{\mathrm{ef}}$ & $4.96^{\mathrm{ef}}$ & $4.62^{\mathrm{e}}$ & $3.95^{\mathrm{f}}$ & $4.57^{\mathrm{d}}$ & $4.26^{t}$ \\
\hline & (25.33) & (32.00) & (28.67) & (17.33) & $(24.00)$ & (20.67) & (14.67) & (20.00) & (17.33) \\
\hline \multirow[t]{2}{*}{$T_{12}$} & $14.31^{\mathrm{a}}$ & $15.43^{\mathrm{a}}$ & $14.87^{\mathrm{a}}$ & $11.74^{\mathrm{a}}$ & $12.45^{\mathrm{a}}$ & $12.10^{\mathrm{a}}$ & $11.10^{\mathrm{a}}$ & $11.81^{\mathrm{a}}$ & $11.46^{\mathrm{a}}$ \\
\hline & $(204.00)$ & (237.33) & (220.67) & (137.33) & $(154.67)$ & (146.00) & (122.67) & (138.67) & (130.67) \\
\hline $\operatorname{Lsd}_{(0.05)}$ & Sig. & Sig. & Sig. & Sig. & Sig. & Sig. & Sig. & Sig. & Sig. \\
\hline
\end{tabular}

*Figures in parentheses are means of original values. Data subjected to transformation $(\sqrt{x+1})$. Treatment means with the letters in common are not significant by DNMRT test at $5 \%$ level of significance

Treatment details- $\mathrm{T}_{1}$ : Pendimethalin PE $750 \mathrm{~g} / \mathrm{ha}, \mathrm{T}_{2}$ : Pendimethalin + metribuzin (Tank mix) PE $(500+175 \mathrm{~g} / \mathrm{ha})$, $\mathrm{T}_{3}$ : Pendimethalin (PE) $500 \mathrm{~g} / \mathrm{ha} f b$ sulfosulfuron (PoE) $18 \mathrm{~g} / \mathrm{ha}\left(\mathrm{T}_{3}\right), \mathrm{T}_{4}$ : Sulfosulfuron PoE (25 g/ha), $\mathrm{T}_{5}$ : Sulfosulfuron + 2,4-D (SS) (Tank mix) PoE (25 + $250 \mathrm{~g} / \mathrm{ha}), \mathrm{T}_{6}$ : Sulfosulfuron + metsulfuron (Premix) PoE $(30+2$ $\mathrm{g} / \mathrm{ha}), \mathrm{T}_{7}$ : Clodinafop PoE (60 g/ha), $\mathrm{T}_{8}$ : Clodinafop + metsulfuron (Premix) PoE $\left(60+4 \mathrm{~g} / \mathrm{ha}\right.$ ), $\mathrm{T}_{9}$ : Metsulfuron methyl PoE (4 g/ha), $\mathrm{T}_{10}$ : Mesosulfuron + iodosulfuron (Premix) PoE $(12+2.4 \mathrm{~g} / \mathrm{ha}), \mathrm{T}_{11}$ : Hand weeding (20 and 40 DAS) and $\mathrm{T}_{12}$ : Un-weeded control 
Table.2 Dry weight of weed as influenced by various weed management practices

\begin{tabular}{|c|c|c|c|c|c|c|c|c|c|c|}
\hline \multirow[t]{3}{*}{ Treatment } & \multicolumn{9}{|c|}{ Dry weight of weed $\left(\mathrm{g} / \mathrm{m}^{2}\right)$} & \multirow{3}{*}{$\begin{array}{l}\text { WCE }(\%) \\
\text { at harvest }\end{array}$} \\
\hline & \multicolumn{3}{|c|}{ At 40 DAS } & \multicolumn{3}{|c|}{ At 80 DAS } & \multicolumn{3}{|c|}{ At harvest } & \\
\hline & 2017-18 & 2018-19 & Pooled & 2017-18 & 2018-19 & Pooled & 2017-18 & 2018-19 & Pooled & \\
\hline \multirow{2}{*}{$T_{1}$} & $4.38^{\mathrm{d}}$ & $4.69^{c}$ & $4.53^{\mathrm{d}}$ & $15.18^{\mathrm{b}}$ & $13.90^{\mathrm{b}}$ & $14.54^{\mathrm{b}}$ & $18.57^{\mathrm{b}}$ & $17.15^{\mathrm{b}}$ & $17.86^{\mathrm{bc}}$ & \multirow[t]{2}{*}{37.93} \\
\hline & $(18.20)$ & $(21.10)$ & $(19.65)$ & $(230.53)$ & (192.67) & $(211.60)$ & $(345.30)$ & $(294.83)$ & $(320.07)$ & \\
\hline \multirow{2}{*}{$\mathbf{T}_{2}$} & $4.43^{d}$ & $4.56^{c}$ & $4.49^{d}$ & $14.08^{\mathrm{bc}}$ & $12.80^{\mathrm{bc}}$ & $13.44^{\mathrm{b}}$ & $17.46^{\mathrm{b}}$ & $15.99^{\mathrm{b}}$ & $16.72^{c}$ & \multirow[t]{2}{*}{45.51} \\
\hline & (18.67) & (19.93) & (19.30) & $(199.60)$ & $(165.13)$ & (182.37) & $(305.67)$ & $(256.40)$ & $(281.03)$ & \\
\hline $\mathbf{T}_{\mathbf{3}}$ & $3.60^{\mathrm{ef}}$ & $3.45^{\mathrm{d}}$ & $3.53^{\mathrm{e}}$ & $8.37^{\mathrm{e}}$ & $7.61^{\mathrm{ef}}$ & $7.99^{\mathrm{ef}}$ & $5.46^{\mathrm{de}}$ & $4.37^{\mathrm{de}}$ & $4.92^{f}$ & 95.42 \\
\hline \multirow{2}{*}{$\mathbf{T}_{4}$} & $4.80^{\mathrm{cd}}$ & $4.35^{\mathrm{c}}$ & $4.58^{\mathrm{d}}$ & $10.70^{d}$ & $9.37^{\mathrm{e}}$ & $10.03^{d}$ & $7.03^{\mathrm{d}}$ & $6.21^{\mathrm{d}}$ & $6.62^{e}$ & \multirow[t]{2}{*}{91.63} \\
\hline & (22.23) & (18.27) & $(20.25)$ & (113.80) & (86.77) & (100.28) & $(48.70)$ & (37.67) & $(43.18)$ & \\
\hline \multirow{2}{*}{$\mathbf{T}_{5}$} & $3.51^{f}$ & $3.17^{\mathrm{d}}$ & $3.34^{\mathrm{e}}$ & $7.79^{\mathrm{e}}$ & $7.00^{f}$ & $7.39^{f}$ & $4.21^{\mathrm{e}}$ & $3.41^{\mathrm{e}}$ & $3.81^{\mathrm{f}}$ & \multirow[t]{2}{*}{97.27} \\
\hline & (11.40) & $(9.20)$ & $(10.30)$ & $(59.70)$ & (48.07) & $(53.88)$ & (17.53) & $(10.70)$ & $(14.12)$ & \\
\hline \multirow{2}{*}{$T_{6}$} & $1.00^{\mathrm{g}}$ & $1.00^{\mathrm{e}}$ & $1.00^{\mathrm{f}}$ & $1.00^{\mathrm{f}}$ & $1.00^{\mathrm{g}}$ & $1.00^{\mathrm{g}}$ & $1.00^{\mathrm{f}}$ & $1.00^{\mathrm{f}}$ & $1.00^{\mathrm{g}}$ & \multirow[t]{2}{*}{100.0} \\
\hline & $(0.00)$ & $(0.00)$ & $(0.00)$ & $(0.00)$ & $(0.00)$ & $(0.00)$ & $(0.00)$ & $(0.00)$ & $(0.00)$ & \\
\hline $\mathbf{T}_{7}$ & $6.34^{\mathrm{b}}$ & $6.40^{\mathrm{b}}$ & $6.37^{b}$ & $12.01^{\mathrm{cd}}$ & $11.51^{\mathrm{cd}}$ & $11.76^{\mathrm{c}}$ & $12.30^{c}$ & $12.50^{c}$ & $12.40^{\mathrm{d}}$ & 70.21 \\
\hline \multirow{2}{*}{$\mathbf{T}_{8}$} & $1.00^{\mathrm{g}}$ & $1.00^{\mathrm{e}}$ & $1.00^{\mathrm{f}}$ & $1.00^{f}$ & $1.00^{\mathrm{g}}$ & $1.00^{\mathrm{g}}$ & $1.00^{\mathrm{f}}$ & $1.00^{\mathrm{f}}$ & $1.00^{\mathrm{g}}$ & \multirow[t]{2}{*}{100.0} \\
\hline & $(0.00)$ & $(0.00)$ & $(0.00)$ & $(0.00)$ & $(0.00)$ & $(0.00)$ & $(0.00)$ & $(0.00)$ & $(0.00)$ & \\
\hline \multirow{2}{*}{$\mathbf{T}_{9}$} & $5.42^{c}$ & $5.73^{b}$ & $5.58^{\mathrm{c}}$ & $15.15^{b}$ & $14.50^{b}$ & $14.83^{b}$ & $18.87^{b}$ & $17.27^{b}$ & $18.07^{b}$ & \multirow[t]{2}{*}{36.44} \\
\hline & (28.53) & (31.93) & $(30.23)$ & (230.87) & (212.23) & $(221.55)$ & $(357.20)$ & $(298.43)$ & $(327.82)$ & \\
\hline \multirow{2}{*}{$T_{10}$} & $1.00^{\mathrm{g}}$ & $1.00^{\mathrm{e}}$ & $1.00^{\mathrm{f}}$ & $1.00^{f}$ & $1.00^{\mathrm{g}}$ & $1.00^{\mathrm{g}}$ & $1.00^{\mathrm{f}}$ & $1.00^{f}$ & $1.00^{\mathrm{g}}$ & \multirow[t]{2}{*}{100.0} \\
\hline & $(0.00)$ & $(0.00)$ & $(0.00)$ & $(0.00)$ & $(0.00)$ & $(0.00)$ & $(0.00)$ & $(0.00)$ & $(0.00)$ & \\
\hline \multirow{2}{*}{$T_{11}$} & $4.25^{\mathrm{de}}$ & $4.50^{c}$ & $4.37^{\mathrm{d}}$ & $8.24^{\mathrm{e}}$ & $9.43^{\mathrm{de}}$ & $8.83^{\mathrm{de}}$ & $6.29^{d}$ & $6.13^{d}$ & $6.21^{\mathrm{e}}$ & \multirow[t]{2}{*}{92.59} \\
\hline & (17.10) & (19.27) & (18.18) & (67.07) & (88.13) & (77.60) & (39.50) & (36.83) & (38.17) & \\
\hline \multirow{2}{*}{$T_{12}$} & $10.35^{\mathrm{a}}$ & $11.21^{\mathrm{a}}$ & $10.78^{a}$ & $19.66^{\mathrm{a}}$ & $19.91^{\mathrm{a}}$ & $19.79^{a}$ & $22.89^{a}$ & $22.53^{a}$ & $22.71^{a}$ & \multirow[t]{2}{*}{ - } \\
\hline & (106.17) & (124.77) & (115.47) & (385.77) & (396.33) & (391.05) & $(523.63)$ & $(506.57)$ & $(515.10)$ & \\
\hline $\operatorname{Lsd}_{(0.05)}$ & Sig. & Sig. & Sig. & Sig. & Sig. & Sig. & Sig. & Sig. & Sig. & - \\
\hline
\end{tabular}

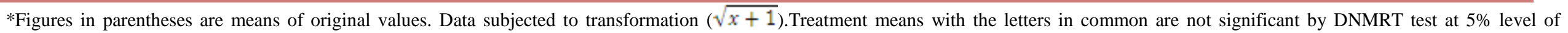
significance

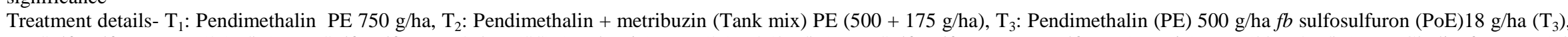

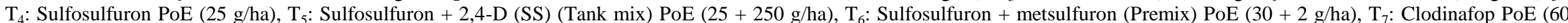

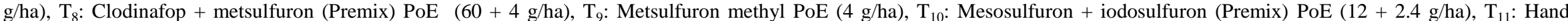
weeding (20 and $40 \mathrm{DAS}$ ) and $\mathrm{T}_{12}$ : Un-weeded control 
Table.3 Yield, weed index and economics as influenced by various weed management practices

\begin{tabular}{|c|c|c|c|c|c|c|c|c|c|c|}
\hline \multirow[t]{3}{*}{ Treatment } & \multirow{2}{*}{\multicolumn{3}{|c|}{ Grain yield (t/ha) }} & \multirow{2}{*}{\multicolumn{3}{|c|}{ Straw yield (t/ha) }} & \multirow{3}{*}{$\begin{array}{c}\text { Weed } \\
\text { Index } \\
(\%)\end{array}$} & \multirow{2}{*}{\multicolumn{3}{|c|}{ (Mean of two year) }} \\
\hline & & & & & & & & & & \\
\hline & 2017-18 & 2018-19 & Pooled & 2017-18 & 2018-19 & Pooled & & $\begin{array}{c}\text { Gross } \\
\text { realiza - } \\
\text { tion } \\
(₹ / h a)\end{array}$ & $\begin{array}{c}\text { Net } \\
\text { realiza - } \\
\text { tion } \\
\text { (₹/ha) }\end{array}$ & $\begin{array}{c}\mathrm{B}: \mathrm{C} \\
\text { ratio }\end{array}$ \\
\hline $\mathbf{T}_{1}$ & $3.63^{d}$ & $3.90^{\mathrm{e}}$ & $3.77^{\mathrm{h}}$ & $5.84^{\mathrm{d}}$ & $6.81^{c}$ & $6.32^{d}$ & 34.96 & 82073 & 48144 & 2.42 \\
\hline $\mathbf{T}_{2}$ & $3.87^{\mathrm{cd}}$ & $4.21^{\mathrm{de}}$ & $4.04^{\mathrm{gh}}$ & $6.57^{\mathrm{bcd}}$ & $7.20^{\mathrm{bc}}$ & $6.89^{\mathrm{cd}}$ & 30.23 & 88245 & 53998 & 2.58 \\
\hline $\mathbf{T}_{3}$ & $4.98^{\mathrm{ab}}$ & $5.61^{\mathrm{ab}}$ & $5.30^{\mathrm{abc}}$ & $7.37^{\mathrm{abc}}$ & $8.40^{\mathrm{ab}}$ & $7.89^{\mathrm{ab}}$ & 8.65 & 114005 & 79042 & 3.26 \\
\hline $\mathbf{T}_{4}$ & $4.37^{\mathrm{bcd}}$ & $4.96^{\mathrm{bcd}}$ & $4.66^{\mathrm{def}}$ & $6.89^{\mathrm{abcd}}$ & $7.59^{\mathrm{abc}}$ & $7.24^{\mathrm{bc}}$ & 19.53 & 100801 & 67132 & 3.00 \\
\hline $\mathbf{T}_{5}$ & $4.73^{\mathrm{abc}}$ & $5.36^{\mathrm{abc}}$ & $5.04^{\text {cde }}$ & $7.08^{\mathrm{abcd}}$ & $8.22^{\mathrm{abc}}$ & $7.65^{\mathrm{abc}}$ & 12.97 & 108767 & 74982 & 3.22 \\
\hline $\mathbf{T}_{6}$ & $5.41^{\mathrm{a}}$ & $5.93^{\mathrm{ab}}$ & $5.67^{\mathrm{ab}}$ & $8.02^{\mathrm{a}}$ & $8.84^{\mathrm{a}}$ & $8.43^{\mathrm{a}}$ & 2.10 & 122029 & 87887 & 3.58 \\
\hline $\mathbf{T}_{7}$ & $4.29^{\mathrm{bcd}}$ & $4.60^{\text {cde }}$ & $4.44^{\mathrm{efg}}$ & $6.83^{b c d}$ & $7.50^{\mathrm{bc}}$ & $7.17^{\mathrm{bcd}}$ & 23.20 & 96420 & 62529 & 2.85 \\
\hline $\mathbf{T}_{8}$ & $5.47^{\mathrm{a}}$ & $6.12^{\mathrm{a}}$ & $5.80^{\mathrm{a}}$ & $8.15^{\mathrm{a}}$ & $8.95^{\mathrm{a}}$ & $8.55^{\mathrm{a}}$ & - & 124641 & 90336 & 3.64 \\
\hline $\mathbf{T}_{9}$ & $4.26^{\mathrm{bcd}}$ & $4.53^{\text {cde }}$ & $4.40^{\mathrm{fg}}$ & $6.40^{\mathrm{cd}}$ & $7.21^{\mathrm{bc}}$ & $6.81^{\mathrm{cd}}$ & 23.96 & 94968 & 61738 & 2.86 \\
\hline $\mathbf{T}_{10}$ & $4.75^{\mathrm{abc}}$ & $5.46^{\mathrm{abc}}$ & $5.11^{\text {bcd }}$ & $7.30^{\mathrm{abc}}$ & $8.65^{\mathrm{ab}}$ & $7.97^{\mathrm{ab}}$ & 11.97 & 110520 & 76228 & 3.22 \\
\hline $\mathbf{T}_{11}$ & $5.30^{\mathrm{a}}$ & $5.97^{\mathrm{a}}$ & $5.64^{\mathrm{abc}}$ & $7.91^{\mathrm{ab}}$ & $8.86^{\mathrm{a}}$ & $8.39^{a}$ & 2.78 & 121338 & 86433 & 3.48 \\
\hline $\mathbf{T}_{12}$ & $1.65^{\mathrm{e}}$ & $1.90^{\mathrm{f}}$ & $1.77^{\mathrm{i}}$ & $3.52^{\mathrm{e}}$ & $4.54^{\mathrm{d}}$ & $4.03^{\mathrm{e}}$ & 69.39 & 40222 & 8164 & 1.26 \\
\hline Lsd $_{(0.05)}$ & Sig. & Sig. & Sig. & Sig. & Sig. & Sig. & 34.96 & 82073 & - & - \\
\hline
\end{tabular}

*Mean followed by common letter (s) in column are not significant by DNMRT test at $5 \%$ level of significance

Treatment details- $\mathrm{T}_{1}$ : Pendimethalin PE $750 \mathrm{~g} / \mathrm{ha}, \mathrm{T}_{2}$ : Pendimethalin + metribuzin (Tank mix) PE $\left(500+175 \mathrm{~g} / \mathrm{ha}\right.$ ), $\mathrm{T}_{3}$ : Pendimethalin (PE) $500 \mathrm{~g} / \mathrm{ha} \mathrm{fb}$ sulfosulfuron (PoE) $18 \mathrm{~g} / \mathrm{ha}\left(\mathrm{T}_{3}\right), \mathrm{T}_{4}$ : Sulfosulfuron PoE (25 g/ha), $\mathrm{T}_{5}$ : Sulfosulfuron + 2,4-D (SS) (Tank mix) PoE (25 + $\left.250 \mathrm{~g} / \mathrm{ha}\right), \mathrm{T}_{6}$ : Sulfosulfuron + metsulfuron (Premix) PoE (30 $+2 \mathrm{~g} / \mathrm{ha}), \mathrm{T}_{7}$ : Clodinafop PoE (60 g/ha), $\mathrm{T}_{8}$ : Clodinafop + metsulfuron (Premix) PoE $(60+4 \mathrm{~g} / \mathrm{ha}), \mathrm{T}_{9}$ : Metsulfuron methyl PoE $(4 \mathrm{~g} / \mathrm{ha}), \mathrm{T}_{10}$ : Mesosulfuron + iodosulfuron (Premix) PoE $(12+2.4 \mathrm{~g} / \mathrm{ha}), \mathrm{T}_{11}$ : Hand weeding (20 and $\left.40 \mathrm{DAS}\right)$ and $\mathrm{T}_{12}$ : Un-weeded control 
Result regarding weed index revealed that application of sulfosulfuron + metsulfuron (PoE) $30+2 \mathrm{~g} / \mathrm{ha}\left(\mathrm{T}_{6}\right)$ followed by hand weeding at 20 and 40 DAS $\left(\mathrm{T}_{11}\right)$ and pendimethalin PE $500 \mathrm{~g} / \mathrm{ha} f b$ sulfosulfuron $(\mathrm{PoE}) 18 \mathrm{~g} / \mathrm{ha}\left(\mathrm{T}_{3}\right)$ recorded lower weed index as compared to rest of treatments. Lower weed index was achieved due to better efficacy of herbicidal treatments against weed thereby least crop weed competition during entire crop season. Among sole application of herbicide, pendimethalin (750 g/ha) applied as pre emergence recorded higher weed index of $34.96 \%$ than that of recorded under metsulfuron methyl PoE (4 g/ha) ( $\left.\mathrm{T}_{9}\right)$, Clodinafop PoE $(60 \mathrm{~g} / \mathrm{ha}) \quad\left(\mathrm{T}_{7}\right)$ and sulfosulfuron PoE (25 g/ha) ( $\left.\mathrm{T}_{4}\right)$. In general, sequential application of pendimethalin $\mathrm{PE}$ $500 \mathrm{~g} / \mathrm{ha} f b$ sulfosulfuron (PoE) $18 \mathrm{~g} / \mathrm{ha}\left(\mathrm{T}_{3}\right)$ performed better than sole application of herbicide with respect to recording lower weed index. The findings are supported by Tiwari et al., (2015), Meena et al., (2019) and Singh et al., (2019).

Premix herbicide is favour on higher economical return because it cut down the application cost as well as labour requirement and helps to getting better yield. Higher gross and net realization were noticed under clodinafop + metsulfuron $(\mathrm{PoE}) 60+4 \mathrm{~g} / \mathrm{ha}$ $\left(\mathrm{T}_{8}\right)$ with benefit cost ratio (3.64) followed by application of pre-mix sulfosulfuron + metsulfuron (PoE) $30+2 \mathrm{~g} / \mathrm{ha}\left(\mathrm{T}_{6}\right)$, hand weeding at 20 and 40 DAS $\left(\mathrm{T}_{11}\right)$ and pendimethalin $500 \mathrm{~g} /$ ha $f b$ sulfosulfuron 18 $\mathrm{g} / \mathrm{ha}\left(\mathrm{T}_{3}\right)$. The lowest gross and net realizations as well as benefit cost ratio (1.26) were registered with un-weeded control treatment $\left(\mathrm{T}_{12}\right)$.

On the basis of two years results it can be concluded that effective weed management, higher yields as well as higher monetary return could be achieved with application of pre-mix herbicide clodinafop + metsulfuron
(PoE) $60+4 \mathrm{~g} /$ ha or pre-mix sulfosulfuron + metsulfuron (PoE) $30+2 \mathrm{~g} /$ ha performed better under middle Gujarat conditions.

\section{References}

Angiras N. N, Kumar S, Rana S. S and Sharma N. 2008. Standardization of dose and time of application of clodinafop-propargyl to manage weeds in wheat. Himachal Journal of Agricultural Research 34(2): 15-18.

Bharat, R. and Kachroo, D. 2010. Bioefficacy of herbicides on weeds in wheat (Triticum aestivum L.) and its residual effect on succeeding cucumber (Cucumis sativus L.). Indian Journal of Agronomy 55(1): 46-50

Chaudhari, D. D., Patel, V. J., Patel, H. K., Aakas Mishra, Patel B. D. and Patel, R. B. 2017. Assessment of pre-mix broad spectrum herbicides for weed management in wheat. Indian Journal of Weed Science 49(1): 33-35

Meena, V., Kaushik, M. L., Dotaniya, Meena, B. P. and Das, H. 2019. Bio-efficacy of readi-mix herbicides on weeds and productivity in late-sown wheat. Indian Journal of Weed Science 51(4): 344-351

Patel B. D., Chaudhari, D. D., Patel, V. J., Patel, H. K., Aakask Mishra, and Parmar, D. J. 2017. Influence of broad spectrum herbicides on yield and complex weed flora of wheat (Triticum aestivum L.). Research on Crops 18(3): 433-437

Rao, A. N. and Nagamani, A. 2010. Integrated Weed Management in IndiaRevisited. Indian Journal of Weed Science 42(3 \& 4): 123-135.

Singh, R. S., Kumar, R., Kumar, M. and Pandey, D. 2019. Effect of herbicides to control weeds in wheat. Indian Journal of Weed Science 51(1): 75-77

Tiwari, A., Kumar, B., Verma, Dev, J. and Raj Kumar. 2015. Bio-efficacy of 
clodinafop-propargyl + metsulfuronmethyl against complex weed flora in wheat. Indian Journal of Weed Science 47(4): 422-424

Yadav Heera Lal. 2017. Comparative Efficiency of New Herbicides in
Managing Resurging Weeds in Wheat and Their Residual Effect on Succeeding Crops. Ph.D. Thesis, Sri Karan Narendra Agriculture University, Jobner.

\section{How to cite this article:}

Vipul Y. Patel, B. D. Patel, V. J. Patel, M. B. Viradiya and Sodavadiya, H. B. 2021. Management of Complex Weed Flora through Herbicide Combinations in Irrigated Wheat (Triticum aestivum L.). Int.J.Curr.Microbiol.App.Sci. 10(01): 3437-3444. doi: https://doi.org/10.20546/ijcmas.2021.1001.405 